\title{
Interaction between voluntary contraction and tonic stretch reflex transmission in normal and spastic patients
}

\author{
PETER D. NEILSON ${ }^{1}$ \\ From the Division of Neurology, the Prince Henry Hospital, and the Schools of \\ Medicine and Physics, University of New South Wales, Sydney, Australia
}

\begin{abstract}
SUMMARY Transmission characteristics of tonic stretch reflex (TSR) pathways in both normal and spastic patients have been measured during different levels of sustained voluntary contraction of the biceps brachii muscle. A cross-correlation technique of analysis was used to separate reflex responses from the total electromyographic activity. TSR transmission in normal subjects was observed to change with the level of voluntary contraction; sensitivity to stretch increased approximately three-fold as the subject stiffened the arm by simultaneously contracting flexor and extensor muscles. In contrast, TSR transmission did not alter during voluntary contraction in spastic patients. It is proposed that in spastic patients supraspinal modulation of reflex transmission is impaired because hypersensitive spinal reflexes short-circuit long loop pathways.
\end{abstract}

Tonic stretch reflexes in man are known chiefly as pathological phenomena, since it is difficult to elicit stretch reflexes in normal man. The limb of a relaxed subject can be moved passively without encountering active resistance. Granit, Kellerth, and Williams (1964) have shown by intracellular recording experiments in the cat that a certain degree of background excitation on alpha and gamma motoneurones is essential for the appearance of stretch reflexes. It would appear that this is also true in man, since stretch reflexes can be activated by the Jendrassik manoeuvre, which is thought to increase bias on gamma and alpha motoneurones (Gassel and Diamantopoulos, 1964).

Merton (1953) speculated that voluntary movement might be initiated by gamma motoneurone activity setting a desired length on the intrafusal muscle fibres. Stretch reflexes were imagined to act like a length follow-up servosystem to contract extrafusal muscle and reduce length error to zero. Stretch reflex pathways which are normally quiescent in a relaxed subject would have to be biased on during voluntary

\footnotetext{
1 Centre Industries Research Scholar.
}

movement initiated by such a proprioceptive servosystem.

It is known that voluntary muscle contractions are supported by alpha and gamma motoneurone coactivation, the so called $\alpha-\gamma$ linkage (Granit, 1966). It has also been shown that when a muscle is lightly contracted the tonic stretch reflex (TSR) of that muscle is activated (Neilson, 1972b). Transmission characteristics of the voluntarily activated TSR in man are more complex than those measured in the decerebrate cat. It was suggested (Neilson, 1972b) that long loop pathways might contribute to the complex TSR response in normal man. Supraspinal control of reflex transmission might adjust the sensitivity of segmental mechanisms to produce an optimum reflex support to voluntary movement. Thus the concomitant activation of stretch reflexes during voluntary contraction might be expected to be a graded control of sensitivity rather than an on-off action. It was decided to test this suggestion by measuring transmission characteristics of TSR pathways of the biceps brachii muscle in normal subjects sustaining different levels of voluntary contraction.

TSR transmission during voluntary contrac- 
tion has also been measured previously in spastic patients (Neilson, 1972c). Responses from these patients were not as complex as those measured in normal subjects. TSR transmission approximated to monosynaptic characteristics measured in the decerebrate cat (Poppele and Terzuolo, 1968; Rosenthal, McKean, Roberts, and Terzuolo, 1970). These findings were interpreted as supporting the proposal that short-circuiting of long loop pathways by overactive spinal reflexes is part of the mechanism of spasticity. If this suggestion is valid, supraspinal control of reflex transmission should be impaired in spastic patients. This conclusion can be tested by measurement of TSR transmission characteristics of biceps brachii muscle in spastic patients sustaining different levels of voluntary contraction.

\section{METHOD}

Two groups of 10 subjects were tested. Individuals with no history of any neurological disturbance comprised the normal group. The second group consisted of 10 cerebral palsied patients who presented a clinical picture of spasticity.

The TSR of a muscle is activated by voluntary contraction of that muscle but the electromyograms (EMGs) of the reflex responses are difficult to measure because they are mixed with potentials caused by the voluntary contraction. A technique to extract the EMG of reflex responses from the total electromyographic activity by cross-correlation analysis between elbow angle and integrated electromyogram (IEMG) from the biceps muscle has previously been described (Neilson, 1972b). It has been shown that voluntary EMG activity is not correlated in a tracking test with joint angle changes of the opposite limb caused by external forces at frequerw cies greater than $2.0 \mathrm{~Hz}$. Normal reaction time limif coherent voluntary responses to less than $2.0 \mathrm{HQg}$ (Neilson, 1972a). Consequently, the component the total EMG activity coherent with joint angter changes caused by disturbance forces at frequencies greater than $2.0 \mathrm{~Hz}$ must be due to involuntary of reflex systems.

The subject lay supine on a couch with the right arm strapped into a frame which constrained move ment of the arm to flexion-extension about the्f elbow. A goniometer attached to the frame recordee elbow angle on a four channel model 5 Grass poly graph. The subject flexed his arm so that the elbow angle was in the $90^{\circ}$ position. Sinusoidal disturbance torques were applied about the elbow joint by spring connected to the arm frame (Fig. 1). The othe $\overrightarrow{\widehat{E}}$ end of the spring was connected to an oscillating cantilever arm on a sinusoidal stretching machinew The frequency of stretching could be varied cors tinuously between 0 and $5.0 \mathrm{~Hz}$. Frequencies were tested in random order at intervals of $0.25 \mathrm{~Hz}$ through this range. The oscillating cantilever arng stretched the spring and therefore increased theo disturbance force applied to the limb. The changesicus elbow angle caused by the force was recorded on the polygraph.

Beckman EMG surface electrodes were attache्fdp $5 \mathrm{~cm}$ apart, over the bellies of the biceps and tricesps muscles. The EMG signals were monitored orn as 17 in. oscilloscope to ensure that they were not cent taminated by movement artefact. The integrated electromyogram of biceps was recorded (TC $=0$ $0.16 \mathrm{sec})$. Amplitude and phase distortion introduced into the IEMG signal by the integrating filter was corrected by multiplying gain and phase by thes

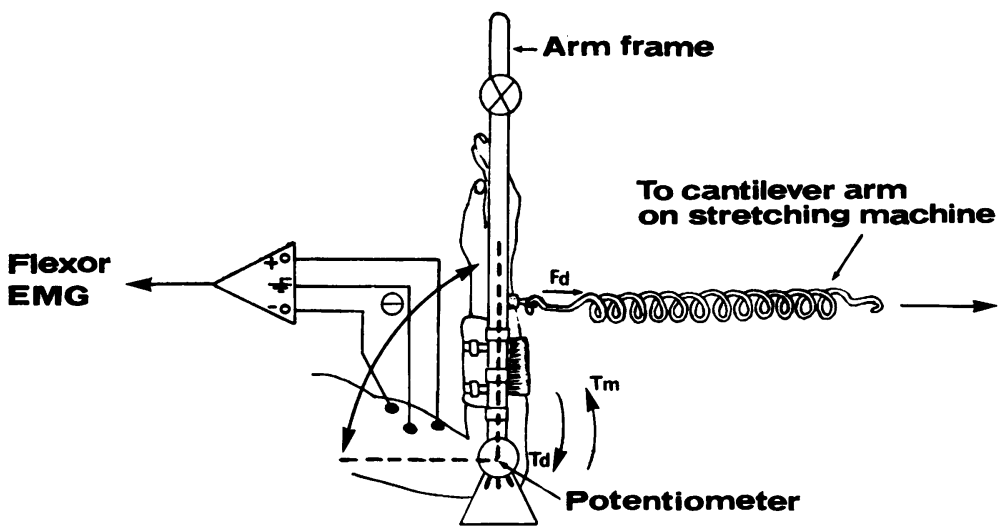

FIG. 1. Diagram illustrating subject's arm strapped in arm frame which constrained movement to flexion-extension about the elbow. Disturbance forces were applied by stretching spring. 
inverse frequency response characteristics of the filter. The IEMG signal from the biceps muscle was calibrated by adjusting the sensitivity of the IEMG amplifier to produce a $25 \mathrm{~mm}$ deflection of the polygraph pen above the baseline while the subject supported a $10 \mathrm{~kg}$ weight attached to a point on the arm frame $30 \mathrm{~cm}$ from the axis of elbow rotation.

During each test instructions were given to stiffen the arm very slowly by simultaneously contracting elbow flexor and extensor muscles. The level of voluntary contraction of the biceps brachii muscle was slowly increased and decreased during each test. The order in which voluntary contraction was increased or decreased as well as the order in which the frequencies were tested was randomized.

Each test lasted two to three minutes. A 5 to 10 minute rest period was given between each test to avoid fatigue.

\section{TECHNIQUES OF ANALYSIS}

The use of two techniques of analysis allowed the results from one technique to be verified by the other.

1. Averaging of IEMG and joint angle responses.
2. Cross-correlation and spectral analysis of IEMG and joint angle signals.

AVERAGING Sinusoidal joint angle and IEMG cycles were numbered consecutively through each test. Individual cycles were selected for measurement by using a table of random numbers. Minimum and maximum values were measured in millimetres as shown in Fig. 2. If the difference between the two minimum values of either the joint angle or the IEMG cycle was greater than $5 \mathrm{~mm}$, all of the data from that cycle were rejected on the grounds that the level of voluntary contraction was changing too rapidly. The average value of the two minimum IEMG measurements provided an indicator for the level of voluntary contraction during that cycle. Using this indicator the data for each cycle were sorted into six levels of voluntary contraction corresponding to six $5 \mathrm{~mm}$ intervals between 0 and $30 \mathrm{~mm}$ of pen deflection. The average peak to peak amplitude was calculated for each joint angle and IEMG cycle. Approximately 100 randomly selected cycles were measured for each level of voluntary contraction at each test frequency. Mean values of

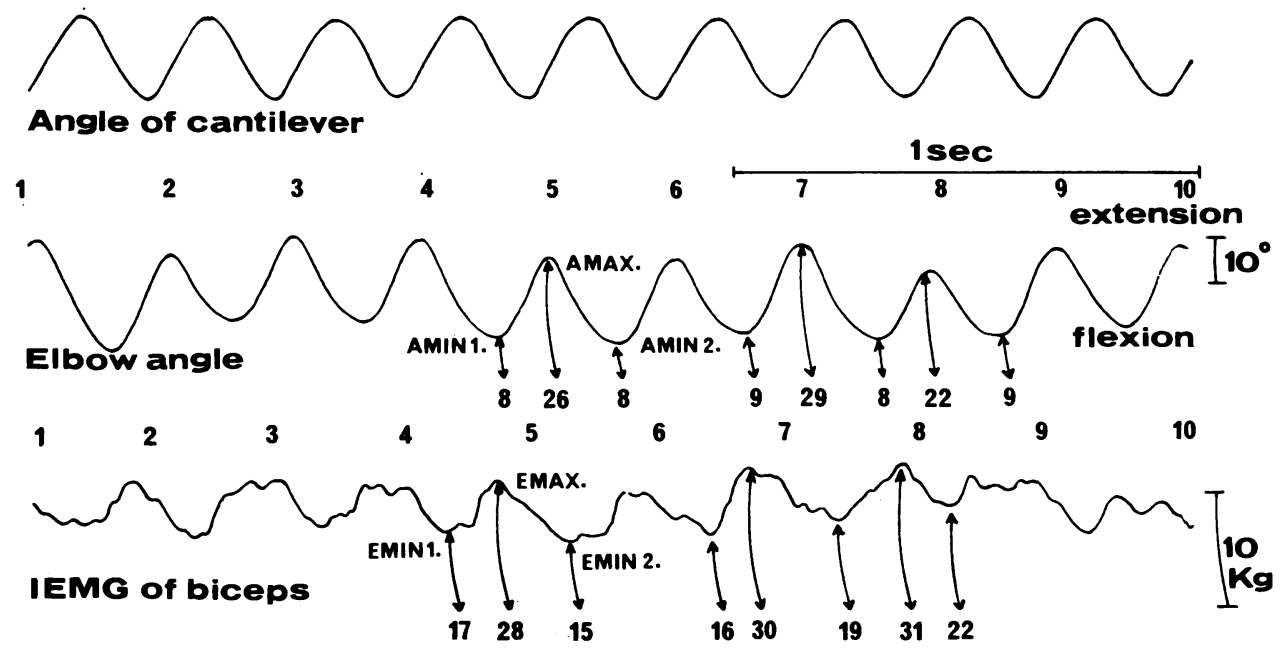

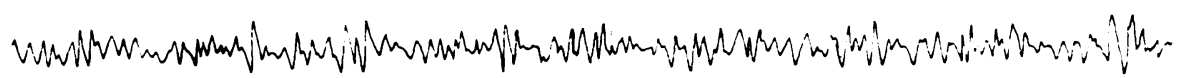

EMG of triceps

FIG. 2. Page of polygraph recording showing how measurements were made to collect data for the averaging technique of analysis. Individual cycles were numbered consecutively and randomly selected for measurement. Minimum angle (AMIN1, AMIN2) minimum integrated electromyogram (EMIN1, EM1N2), maximum angle $(A M A X)$ and maximum integrated electromyogram (EMAX) were measured as shown. 
the peak to peak amplitude of the sinusoidal joint angle movement, peak to peak amplitude of sinusoidal IEMG changes and gain (defined as the ratio of IEMG amplitude to change in joint angle) were calculated for each level of voluntary contraction and frequency. Gain was plotted against the level of voluntary contraction for each frequency tested. The gain curves included correction for distortion by the integrating filter.

A measure of statistical significance of the change in gain between the lowest and highest levels of voluntary contraction was obtained by a Student $t$ test.

CROSS-CORRELATION AND SPECTRAL ANALYSIS This technique has been described previously (Neilson, 1972b). Joint angle and IEMG signals were digitalized by measuring the deflection above a baseline on the polygraph at a rate of either 10 or $20 / \mathrm{sec}$ (Fig. 3). Average deflection of IEMG above the baseline during each $1 \mathrm{sec}$ interval was computed and used as a measure of voluntary contraction during that interval. Angle and IEMG data collected during each $1 \mathrm{sec}$ interval were sorted into six levels of voluntary contraction corresponding to six $5 \mathrm{~mm}$ increments of average IEMG deflection between 5 and $35 \mathrm{~mm}$. Correlation functions and power spectra were calculated using the method described by Jenkins and Watts (1968). Gain and phase shift were calculated at each level of voluntary contraction at each frequency tested. A correction was made for the distortion in the IEMG signal caused by the integrating filter.
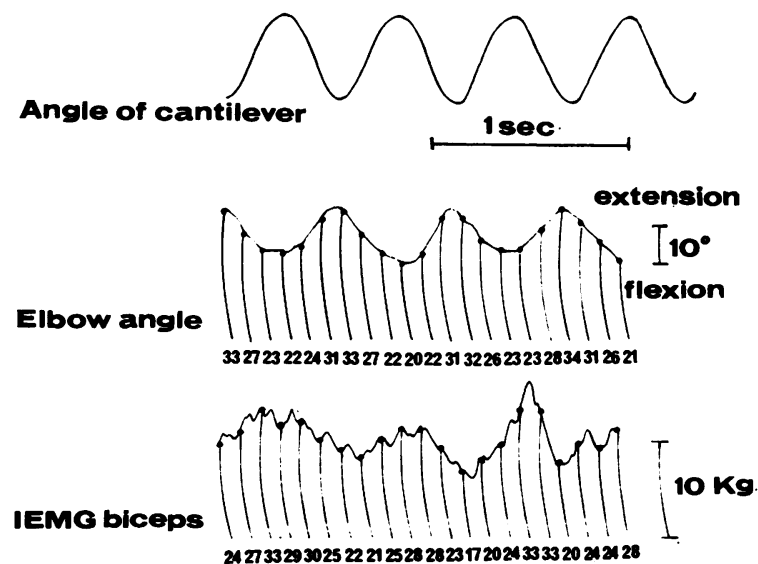

EMG triceps

\section{RESULTS}

The magnitude of the IEMG responses to $\sin x$ soidal stretching increased with voluntary cors traction in all normal subjects tested (Fig. 4. Gain of TSR pathways (ratio of the amplitude of sinusoidal variation of IEMG to amplitude of sinusoidal joint angle movement) increased with the level of voluntary contraction for all fret. quencies above $2.0 \mathrm{~Hz}$ (Fig. 5). The increase gain between the lowest and highest levels of voluntary contraction was always found to be statistically significant beyond $0 \cdot 1 \%$ level.

Amplitude of IEMG responses to sinusoid武 stretching did not vary with voluntary contra tion in the spastic patients (Fig. 6). Gain of TSR in spastic patients was high even at low levels of voluntary contraction and, if anything, tended to decrease with increasing contraction. Gaint has been plotted against level of voluntary cone traction and contrasted with the variation i gain observed in normal subjects (Fig. 7\% Student $t$ testing confirmed that the differences in gain between the lowest and highest levels-of voluntary contraction in spastic patients was â statistically significant at any frequency.

EMG reflex responses were advanced in phise ahead of biceps stretch in both spastic normal subjects. For spastic patients, howeven the phase lead did not change significantly voluntary contraction, whereas in normal s,
FIG. 3. Page of polygraph recording showing how measurements were made to collect data for cross-correlation technique $\supset$ of analysis. Deflections of both elbow ang tracing and integrated electromyogram above the baseline were measured in millimetres at a sampling rate equivalent to either 10 or 20 samples per second through. out the full length of the test. Approximately 1,000 points were sampled at each level of contraction at each frequency. 


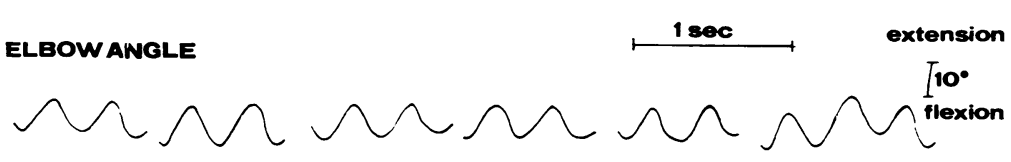

FIG. 4. IEMG responses to sinusoidal stretching at increasing levels of voluntary contraction in a normal subject. The average level of the

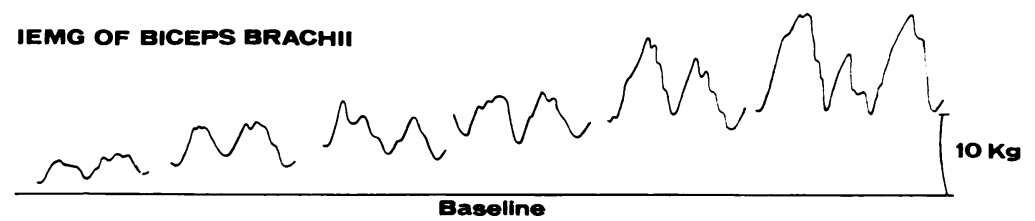
integrated electromyogram above the baseline provides a measure of the level of voluntary contraction. $25 \mathrm{~mm}$ deflection corresponds to $10 \mathrm{~kg}$ supported at the wrist. Sensitivity of the stretch reflex increases with voluntary contraction.

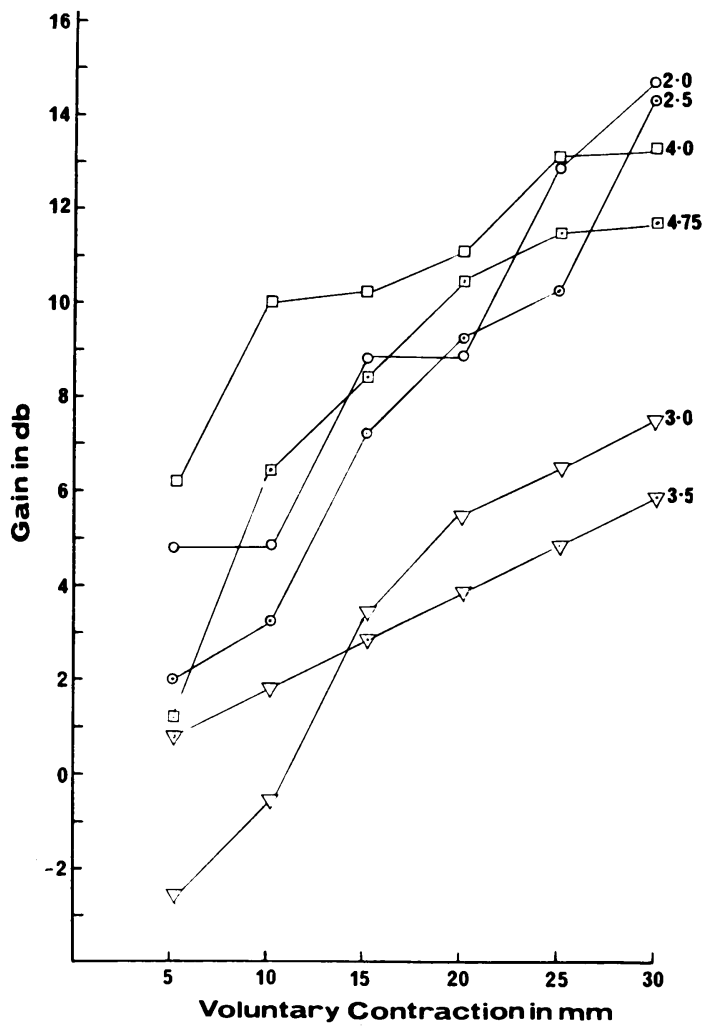

FIG. 5. Gain of TSR plotted against level of voluntary contraction for a normal subject. A family of curves has been plotted for different frequencies of stretching showing that the rate of increase in gain with contraction depends upon frequency. Voluntary contraction is measured by the average deflection in $\mathrm{mm}$ of the integrated electromyogram above the baseline. IEMG has been calibrated so that $25 \mathrm{~mm}$ deflection is equivalent to $10 \mathrm{~kg}$ weight supported at the wrist. The increase in gain between the lowest and highest levels of contraction was significant (Student $\mathrm{t}$ test) beyond $0 \cdot 1 \%$ level. jects it increased by as much as $90^{\circ}$ at some frequencies $(2 \cdot 0$ to $3.0 \mathrm{~Hz})$.

Gain vs frequency characteristics of normal subjects contained resonant peaks at each level of voluntary contraction confirming the finding reported previously (Neilson, 1972b). The shape of the frequency response curves changed with the level of voluntary contraction, whereas the frequency response curves from spastic patients contained no peaks of resonance nor did they vary significantly with voluntary contraction (Fig. 8).

\section{DISCUSSION}

The TSR of biceps muscle in normal subjects is activated during voluntary contraction. Activation, however, is not a simple on-off action. TSR transmission characteristics vary with contraction in a complex manner. At any one frequency of stretch the gain of TSR transmission increases with the level of contraction but the rate of increase varies from frequency to frequency. This causes the shape of the gain $v s$ frequency curve to alter as the muscle is voluntarily contracted. The size and location of the resonant peaks vary as the subject stiffens the arm and therefore the shape of the reflex response to a transient stretch applied to the muscle can also be expected to vary.

During a voluntary movement the higher motor centres presumably signal commands not only to control lengths and tensions of groups of muscles but also to set the gains of the reflex pathways. It is proposed that descending activity determines the sensitivity, timing, and shape of 


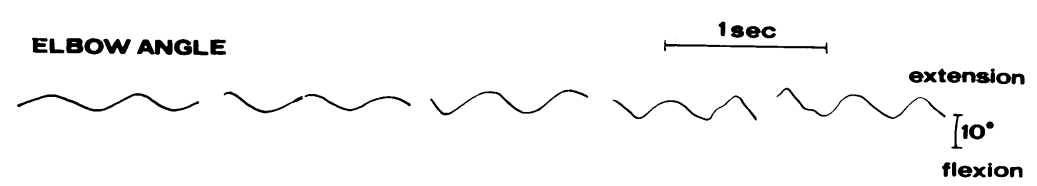

FIG. 6. IEMG responses to sinusoidal stretching at increasing levels of voluntary contraction in a spastic patient. Level of voluntary contraction increases from IEMG OF BICEPS BRACHII left to right. An average IEMG deflection of $25 \mathrm{~mm}$ corresponds to $10 \mathrm{~kg}$ sup-

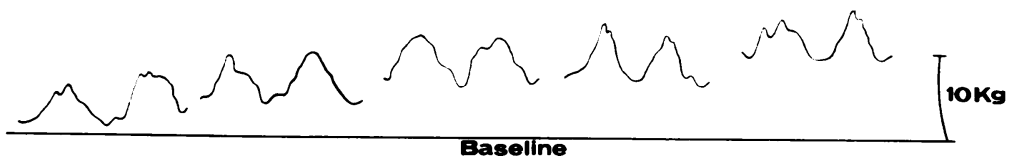
ported at the wrist. Reflex sensitivity does not increase with contraction level.

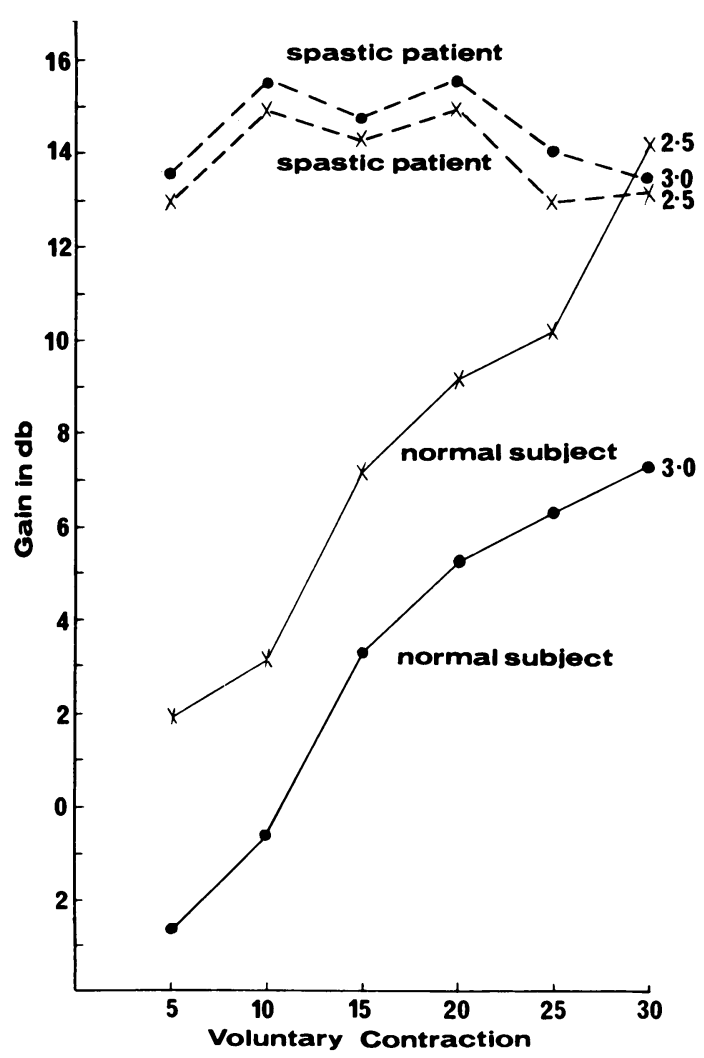

FIG. 7. Gain of TSR plotted against level of voluntary contraction at two different frequencies of stretching $(2.5$ and $3.0 \mathrm{~Hz})$. Curves for a spastic patient have been contrasted with those of a normal subject. For the spastic patient reflex gain is high at low levels of contraction but does not change significantly with increasing levels of voluntary contraction. the TSR responses as well as the initiation of movement. This proposition is not without support from experiment. Synaptic transmission of the reflex connections of groups Ib, II, and III muscle afferent nerve fibres in the cat hindlimb can be inhibited by brain-stem stimulation (Holmqvist and Lundberg, 1959) and both alpha and gamma efferent nerve fibres can be influenced by vestibular activity (Andersson and Gernandt, 1956). Higgins (1968) demonstrate 6 o control of triceps surae muscle stretch refle characteristics by vestibular activity in acutel $\overrightarrow{\mathbb{D}}$ decerebrate cats and stimulation of the intact

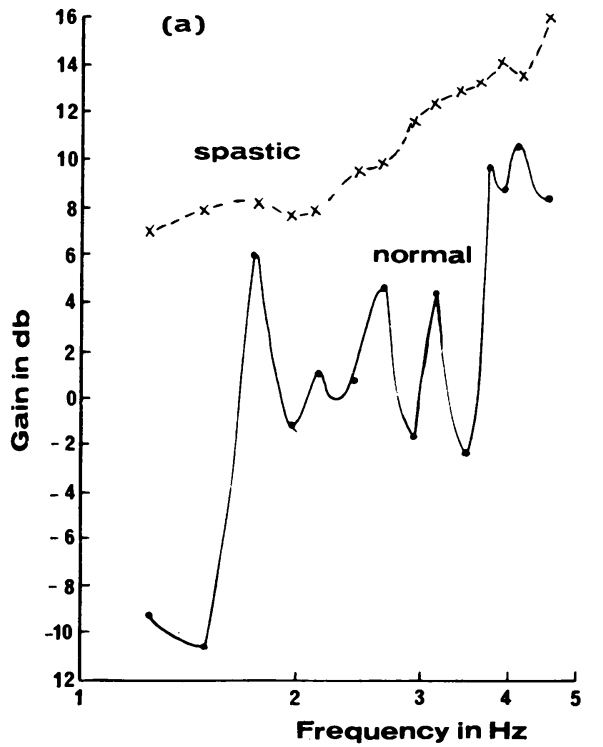

Fig. 8(a) 


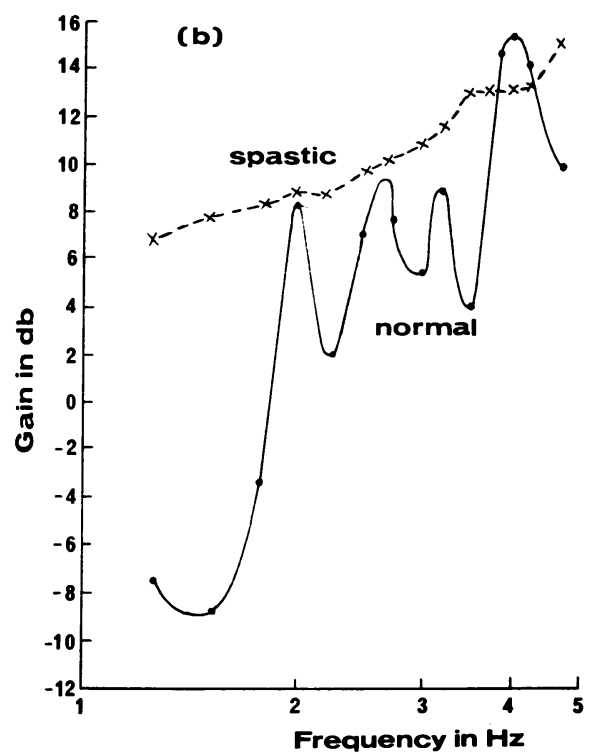

(b)

FIG. 8. Gain of TSR of spastic patient and normal subject plotted against frequency of stretching. (a) Data collected during a sustained voluntary contraction causing a mean IEMG deflection of $10 \mathrm{~mm}$ equivalent to $4.5 \mathrm{~kg}$ supported at the wrist. (b) Data collected during a sustained voluntary contraction equivalent to $9 \mathrm{~kg}$ supported at the wrist. Curves illustrate increase in gain and change in shape of resonant peaks with voluntary contraction in normal subject, while no significant change occurs in spastic patient.

cerebellum has been shown to add a dynamic modification to the myotatic response (Higgins, Partridge, and Glaser, 1962). Stimulation of the pontine and medullary reticular formation facilitates and inhibits respectively the tonic vibration reflex (TVR) of the gastrocnemiussoleus muscle in the decerebrate cat (Gillies, Burke, and Lance, 1971). The TVR is initiated by vibration excitation of primary muscle spindle endings. TVR in normal man can be suppressed completely by voluntary effort (De Gail, Lance, and Neilson, 1966; Eklund and Hagbarth, 1965; Marsden, Meadows, and Hodgson, 1969) but this ability is lost in spastic patients (Hagbarth and Eklund, 1968). Hammond (1956) observed that spinal polysynaptic stretch reflex responses in man can be inhibited completely or preset by descending activity. A study of H-reflex excitability during voluntary contraction of gastrocnemius-soleus and anterior tibial muscles in man during foot pressure tracking allowed Gottlieb, Agarwal, and Stark (1970) to demonstrate that the agonist myotatic loop gain is turned up before movement and the antagonist circuit is turned off to prevent reflex opposition. They found evidence for the existence of a mechanism for the independent regulation of the gain of the monosynaptic reflex arc mediated centrally within the cord.

The complicated shape of the frequency response curves measured in normal subjects emphasizes that the TSR signals are transmitted by neuronal circuits which are sufficiently complex to transform the shape of the response. The total IEMG response may well be composed of the sum of responses from a number of parallel reflexes. At one extreme, corrections for slowly changing loads on a limb can be mediated by long pathways to higher centres where muscle contractions can be consciously activated to oppose the load. At the other extreme, a rapid change of load can induce fast corrections by exciting monosynaptic or short segmental reflex circuits. A number of reflex pathways probably exist between these two extremes which correct for disturbances of intermediate speeds. Interaction between such parallel pathways could produce the complex frequency response curves measured. Furthermore, supraspinal control of the sensitivity of each pathway could cause the change in shape of the frequency response curves observed during changes of voluntary contraction.

Gain and phase curves did not alter with the level of voluntary contraction in spastic patients. The TSR was active even during very low levels of contraction and seemed to be independent of the voluntary control exerted by the patient. In spastic patients a lack of correlation was observed between voluntary and reflex contraction of a muscle. This could lead to a situation in which the reflexes do not support purposive movements but rather compete with them.

As reported previously and verified in this study the transmission characteristics of TSR pathways in spastic patients are not as complex as those measured in normal subjects under similar test conditions. The absence of peaks in 
the gain curves and the smaller phase advancement of IEMG responses ahead of stretch are consistent with the hypothesis that exaggerated sensitivity of spinal reflexes in spasticity causes them to dominate or to short-circuit long loop pathways. Moreover, the failure of the frequency response curves to change shape as the patient increased voluntary contraction supports the concept that the change of gain in normal subjects is caused, at least in part, by supraspinal control of long loop pathways. In spasticity, the gain of the reflex loop appears to be set at a nearmaximal level so that reinforcement by voluntary contraction is unable to cause any increment in gain.

I wish to thank Associate Professor J. W. Lance and Professor E. P. George for their valued advice and encouragement and for their generous provision of equipment from the Schools of Medicine and Physics. The figures were drawn by Miss Susan Casey and prepared by the Department of Medical Illustration, University of New South Wales. The Spastic Centre of N.S.W. provided the Centre Industries Research Scholarship in support of this project.

\section{REFERENCES}

Andersson, S., and Gernandt, B. E. (1956). Ventral root discharge in response to vestibular and proprioceptive stimulation. Journal of Neurophysiology, 19, 524-543.

De Gail, P., Lance, J. W., and Neilson, P. D. (1966). Differential effects on tonic and phasic reflex mechanisms produced by vibration of muscles in man. Journal of Neurology, Neurosurgery, and Psychiatry, 29, 1-11.

Eklund, G., and Hagbarth, K.-E. (1965). Motor effects of vibratory muscle stimuli in man. Electroencephalography and Clinical Neurophysiology, 19, 619.

Gassel, M. M., and Diamantopoulos, E. (1964). The pattern of reinforcement of monosynaptic reflexes in normal subjects and patients with spasticity or rigidity. Neurology (Minneapolis), 14, 555-560.

Gillies, J. D., Burke, D. J., and Lance, J. W. (1971). Supraspinal control of tonic vibration reflex. Journal of Neurophysiology, 34, 302-309.

Gottlieb, G. L., Agarwal, G. C., and Stark, L. (1970). Interactions between voluntary and postural mechanisms of the human motor system. Journal of Neurophysiology, 33, 365381.

Granit, R. (1966). Effects of stretch and contraction on the membrane of motoneurones, pp. 37-50. In Muscular Afferents and Motor Control. Proceedings of the First Nobel Symposium, 1965, Södergarn. Edited by R. Granit. Almqvist and Wiksell: Stockholm.

Granit, R., Kellerth, J.-O., and Williams, T. D. (1964). 'Adjacent' and 'remote' post-synaptic inhibition in motoneurones stimulated by muscle stretch. Journal of Physiology, 174, 453-472.

Hagbarth, K.-E., and Eklund, G. (1968). The effects of muscle vibration in spasticity, rigidity, and cerebellar disorders. Journal of Neurology, Neurosurgery, and Psychiatry, 31, 207-213.

Hammond, P. H. (1956). The influence of prior instruction to the subject on an apparently involuntary neuro-muscular response. Journal of Physiology, 132, P17-18.

Higgins, D. C. (1968). Vestibular component in dynamic muscle stretch. American Journal of Physiology, 214, 482487.

Higgins, D. C., Partridge, L. D., and Glaser, G. H. (1962). A $\vec{\circ}$ transient cerebellar influence on stretch responses. Journal of Neurophysiology, 25, 684-692.

Holmqvist, B., and Lundberg, A. (1959). On the organization of the supraspinal inhibitory control of interneurones of various spinal reflex arcs. Archives Italiennesde Biologie, 97, 340-356.

Jenkins, G. M., and Watts, D. G. (1968). Spectral Analysis and its Applications. Holden-Day: San Francisco.

Marsden, C. D. Meadows, J. C., and Hodgson, H. J. Fo (1969). Observations on the reflex response to musclo $\omega$ vibration and its voluntary control. Brain, 92, 829-846.

Merton, P. A. (1953). Speculations on the servo-control of movement. In The Spinal Cord: a CIBA Foundation Symposium, pp. 247-260. Edited by J. L. Malcolm and $\mathbb{D}$ J. A. B. Gray. Churchill: London.

Neilson, P. D. (1972a). Speed of response or bandwidth ơ voluntary system controlling elbow position in intact manc. Medical and Biological Engineering, 10, 450-459.

Neilson, P. D. (1972b). Frequency response characteristics of the tonic stretch reflexes of biceps brachii muscle in intact $N$ man. Medical and Biological Engineering, 10, 460-472.

Neilson, P. D. (1972c). Voluntary and reflex control of the biceps brachii muscle in spastic-athetotic patients. Journa of Neurology, Neurosurgery, and Psychiatry. (In press.)

Poppele, R. E., and Terzuolo, C. A. (1968). Myotatic reflex : its input-output relation. Science, 159, 743-745.

Rosenthal, N. P., McKean, T. A., Roberts, W. J., and Terzuolo, C. A. (1970). Frequency analysis of stretch reflex and its main subsystems in triceps surae muscles of the cat Journal of Neurophysiology, 33, 713-749. 